\title{
Recent applications of ATR FTIR spectroscopy and imaging to proteins
}

\author{
Stefanie E. Glassford ${ }^{\mathrm{a}}$, Bernadette Byrne ${ }^{\mathrm{b}}$, Sergei G. Kazarian ${ }^{\mathrm{a},{ }^{*}}$ \\ ${ }^{a}$ Department of Chemical Engineering, Imperial College London, SW7 2AZ, U.K. \\ ${ }^{\mathrm{b}}$ Department of Molecular Bioscience, Imperial College London, SW7 2AZ, U.K. \\ "Corresponding author. E-mail address: s.kazarian@imperial.ac.uk
}

\begin{abstract}
Attenuated Total Reflection (ATR) Fourier Transform Infrared (FTIR) spectroscopy is a label-free, non-destructive analytical technique that can be used extensively to study a wide variety of different molecules in a range of different conditions. The aim of this review is to discuss and highlight the recent advances in the applications of ATR FTIR spectroscopic imaging to proteins. It briefly covers the basic principles of ATR FTIR spectroscopy and ATR FTIR spectroscopic imaging as well as their advantages to the study of proteins compared to other techniques and other forms of FTIR spectroscopy. It will then go on to examine the advances that have been made within the field over the last several years, particularly the use of ATR FTIR spectroscopy for the understanding and development of protein interaction with surfaces. Additionally, the growing potential of Surface Enhanced Infrared spectroscopy (SEIRAS) within this area of applications will be discussed. The review includes the applications of ATR FTIR imaging to protein crystallization and for highthroughput studies, highlighting the future potential of the technology within the field of protein structural studies and beyond.
\end{abstract}

Keywords: FT-IR spectroscopy, Chemical imaging, Surface-enhanced spectroscopy, Protein analysis, Total internal reflection, High throughput. 


\section{Introduction}

Our understanding of protein structure and function has advanced dramatically over the last 20 years or so at least in part as a result of the time and effort spent on structural proteomic projects [1]. Obtaining high-resolution 3D protein structures is essential to gain insights into the mechanism of action of these important molecules, as well as to provide a potential framework for rational and targeted drug design. $\mathrm{X}$-ray diffraction is the most commonly used method for determination of high resolution structures but obtaining well-diffracting protein crystals can be both difficult and time consuming [2]. As more and more structures are solved the remaining uncharacterised proteins tend to be those that are the most challenging to work with including multi-subunit complexes, unstructured proteins [3] and integral membrane proteins [4]. Methods to provide efficient highthroughput screening of conditions likely to yield diffracting protein crystals would greatly facilitate studies on the more challenging protein molecules. In addition although $\mathrm{X}$-ray crystallographic studies can reveal much, such studies produce static snapshots of protein molecules and lack dynamic information key to understand the conformation changes associated with function.

Many of the most challenging types of proteins are prone to aggregation and unfolding as a result of the conditions used for isolation and final preparation [5]. Similar problems are known to occur in the case of biopharmaceuticals such as therapeutic antibodies and antibody fragments [6]. Whilst it is likely that increases in protein concentration can result in protein aggregation, the effects of the surfaces that proteins encounter during isolation are far less well understood $[7,8]$. A much better understanding of the effects of surfaces on protein behaviour has major implications for the development of biomedical materials, clinical diagnostics and cellular adhesion $[9,10,11]$. One prominent field is the study of the effect of surface properties on proteins which has major implications in the development of medical devices and implants $[9,12]$. The ability to understand the role that a particular surface has on a proteins conformation, in situ would be extremely valuable.

Infrared spectroscopy, in particular attenuated total reflection (ATR) Fourier transform infrared (FTIR) spectroscopy, has significant potential as an analytical technique to facilitate both protein crystallisation studies and investigations on the effect of surface properties on protein behaviour as well as other key questions related to protein structure and function. This review focuses on the most recent advances in the field of ATR FTIR spectroscopy to proteins, particularly the development of ATR FTIR spectroscopic imaging and surface enhanced infrared absorption spectroscopy.

\section{ATR FTIR spectroscopy}

There are numerous reviews detailing Infrared spectroscopy, therefore only the fundamentals will be discussed here. Infrared spectroscopy is the study of the interaction of infrared light with matter. When a beam of infrared light is directed at a sample, the wavelengths absorbed are dependent on the molecular vibrations of the substance. As such, based on the infrared absorbance of a sample one can determine both chemical and structural information from a sample. The multiple ways in which infrared light can be directed at samples and the range of detectors available allows for the analysis of a wide variety of samples in a range of conditions. 
The most traditional method is transmission, where infrared light is passed through a sample sandwiched between two IR transparent windows. However, since the beam is travelling through the absorbing sample, this method requires short pathlengths of typically no more than $6-10 \mu \mathrm{m}$ between the IR transparent windows, in the case of aqueous samples. This is particularly important when aqueous samples are to be studied because water is a strong absorber of IR light; too long a pathlength would result in all the IR radiation being absorbed by the sample and none reaching the detector. Reflectance infrared spectroscopy is another method where IR light is reflected off the external surface of a sample, it is similar to attenuated total reflection except the infrared beam is not directed through an infrared transparent medium to the sample.

ATR FTIR spectroscopy involves directing the infrared light at an interface between an infrared transparent material with a high refractive index called the internal reflection element (IRE, e.g. a prism made of $\mathrm{ZnSe}$, diamond, silicone or germanium) and a sample on the surface of the IRE. The angle of incidence of the IR beam is greater than the critical angle and as such, total internal reflection occurs. At the reflecting surface, a standing wave of radiation called an evanescent wave is established and it is this evanescent wave that interacts with the sample attenuating the infrared beam of light exiting the IRE. The depth to which the evanescent wave penetrates a sample is defined by the depth of penetration, the depth at which the evanescent wave is attenuated to $1 / \mathrm{e}$, shown in Equation 1. This is dependent on the angle of incidence and the refractive indices of both the prism and the sample. It is typically in the range of $0.5-2 \mu \mathrm{m}$ although it should be noted that absorbance information is obtained from the sample beyond this range, as the evanescent wave probes deeper than the depth of penetration[13]. Since ATR FTIR is inherently a surface layer technique in that the evanescent wave only interacts with the several micrometer thick surface layer of the sample, it has advantages for protein studies in particular. In comparison with transmission, it is much more amenable to the study of aqueous samples in particular and the sample preparation is generally much simpler.

$$
\mathrm{d}_{\mathrm{p}}=\frac{\lambda}{2 \pi \mathrm{n}_{1}\left(\sin ^{2} \theta-\left(\frac{\mathrm{n}_{2}}{\mathrm{n}_{1}}\right)^{2}\right)^{1 / 2}}
$$

(Equation 1)

\section{FTIR spectra of proteins - the amide bands}

The use of IR spectroscopy to gain insight into protein structure and behaviour has been continually developed since the first work on characterising the spectral features of different polypeptide secondary structures and amino acids $[14,15,16]$. The infrared spectra of protein exhibit 5 characteristic bands, three of which are known as amide bands. These are most commonly used in infrared protein studies. The Amide I band, which has the strongest absorption of infrared light, is found between $1600-1700 \mathrm{~cm}^{-1}$. It is primarily caused by stretching vibrations of $\mathrm{C}=\mathrm{O}$ coupled weakly with $\mathrm{C}-\mathrm{N}$ stretch and $\mathrm{N}-\mathrm{H}$ bending. The exact band position is determined by the backbone conformation and the hydrogen bonding pattern within the protein molecule [17]. The Amide II band occurs at $1500-1600 \mathrm{~cm}^{-1}$ and is mainly derived from the $\mathrm{C}-\mathrm{N}$ stretch along with $\mathrm{N}-\mathrm{H}$ in-plane bending. Lastly, the Amide III band is found at $1200-1300 \mathrm{~cm}^{-1}$. The vibrations responsible for this band are a complex mix of $\mathrm{N}-\mathrm{H}$ bending and $\mathrm{C}-\mathrm{N}$ stretching along with deformation vibrations of $\mathrm{C}-\mathrm{H}$ and $\mathrm{N}-\mathrm{H}[17,18,19]$. 
Although all three bands can be useful to elucidate the secondary structure of a protein, the Amide I band is the most sensitive to structural changes and is the most commonly used in secondary structure analysis. Factors responsible for the conformational sensitivity of the Amide I band include hydrogen bonding and coupling between transition dipoles, both inter and intra molecular, which will impact the way in which a protein molecule interacts with IR light [20]. From the study of simple homopolypeptides of known single structure, the relationship between Amide I band position and structure has been proposed allowing predictions about the secondary structure of proteins to be made from IR spectra $[16,18]$. Further analysis of the Amide 1 band is required in order to obtain the types and amounts of secondary structure present; this is achieved through Fourier deconvolution and second derivative, curve fitting and spectral subtraction, for example. Multivariate analysis and other statistical methods are also increasingly being applied to the analysis of protein spectra because of their usefulness in interpreting the wealth and complexity of information contained within the spectra $[21,22,23]$.

There are many examples in the literature of the assignment of protein secondary structure based on the analysis of the Amide I band $[15,16,17,20,24]$. In general, $\alpha$-helical structures have a band peak at wavenumbers $1650-1658 \mathrm{~cm}^{-1} ; \beta$-sheet structures tend to have bands between 1620-1640 $\mathrm{cm}^{-1}$ and between $1670-1695 \mathrm{~cm}^{-1}$; random coil structures occur at around $1644 \mathrm{~cm}^{-1}$ [20]. It is even possible to assign more complex structures such as an $\alpha$-helix overlapped with random coil which can absorbs IR radiation at $1654-1656 \mathrm{~cm}^{-1}$ [17]. Figure 1 shows an example of a protein spectrum, the inset shows curve fitting of the Amide I band for different structural components [19].

The study of aqueous protein samples is challenging because of the overlap of the water bending mode with the Amide 1 band at $1600 \mathrm{~cm}^{-1}$. This could be overcome through the use of $D_{2} \mathrm{O}$; however this can affect the native secondary structure of the protein. Current FTIR spectrometers allow for accurate subtraction of the water band, although care should be taken to avoid over subtraction. There are several papers with guidelines for this procedure but it is widely accepted that the integrity of protein spectral data can be maintained by meeting two subtraction criteria; accurate subtraction of water vapour bands between $1800-1500 \mathrm{~cm}^{-1}$ and ensuring a straight baseline between $2000-1750 \mathrm{~cm}^{-1}$ upon water subtraction [24,25].

\section{ATR FTIR spectroscopy of surfaces and proteins}

The study of the interaction of proteins with different surfaces is important for the design of biomaterials, biomedical devices and for the production of biopharmaceuticals $[10,26,27,28]$. Both the surface chemistry and topography can play a major role in the behaviour of biological materials [29]. As described above, ATR FTIR spectroscopy is a surface layer technique where the absorbance is measured only from the portion of a sample in direct contact with the ATR surface rather than from the bulk. The surface of an IRE (or ATR crystal) can be easily modified making it well placed to study the effect of different surfaces on proteins without the need for additional labelling [11]. It can also easily be coupled with other experimental methods designed to induce changes within proteins such as photochemistry, heat and electrochemistry [30]. Together with the variety of ATR crystals made from different materials available, this provides many opportunities to study the effect of surfaces on the sample. The sensitivity of measurements could potentially be increased by making use of multiple reflection objectives; this increases the pathlength of the measurement [31,32]. However, multiple reflection objectives cannot be used in FTIR imaging because absorbances are 
measured at several different locations. This would create overlap in the images which are based on absorbance values meaning for FTIR imaging single reflection objectives are required [13].

There have been several investigations carried out using ATR FTIR spectroscopy to study the effect of polyelectrolyte multilayers on protein adsorption and on proteins within the layers [33,34,35]. Figure 2 demonstrates the usefulness of ATR penetration depth for examining the multilayer. BSA is adsorbed only into the positively charged layer and as such will absorb infrared. Heat can be applied to the multilayer and the stabilising effect on the protein against heat induced structural changes can be followed by examining the secondary structure from the amide I band [33].

Difference spectroscopy is a key tool in the analysis of proteins using ATR-FTIR spectroscopy. Whereas absorption spectra can provide global information about the sample such as secondary structure, difference spectroscopy can allow small changes in functional groups, hydrogen bonding changes and chemical reactions to be followed in situ using ATR-FTIR spectroscopy [31,32]. This allows for sensitive measurements to be made following subtle changes in protein conformation and can be used to provide details of mechanisms of molecular reactions. One method, demonstrated by Pinkerneil et al. shows the advantages of immobilizing polyhistidine tagged proteins onto the surface of a multiple reflection ATR crystal by first preparing a modified lipid bilayer containing nitrilotriacetic acid (NTA) groups on the surface [31]. Such immobilization is based on established immobilized ion metal affinity chromatography where the selective binding of proteins is activated by the formation of transition metal ion complexes [32]. Once proteins are immobilized on the surface, reactions induced by changes in heat, $\mathrm{pH}$ for example, can be followed in situ by difference spectroscopy. This could be potentially applied to any polyhistidine tagged protein of interest.

A study by Onodera et al. demonstrated the ability of ATR FTIR spectroscopy to explore proteinprotein interactions by immobilizing antigens on a functionalised GaAS ATR element [36]. The selective binding of a specific antibody can then be followed in comparison with a non-specific antibody by comparing the secondary structures of the adsorbed proteins from the second derivative spectra of the Amide I band. It was shown that the selective binding of IgG to the antigen sensing layer resulted in spectra high in $\beta$-sheet content, consistent with known IgG structure [36]. However, the spectra of the non specific protein showed no significant amide peaks allowing the differences in specific and non specific binding to be distinguished.

Opportunities also exist to examine protein reactions and behaviour by functionalizing germanium or silicon ATR elements $[37,38]$. Schartner et al. have shown that in combination with difference spectroscopy and by using multiple reflection IREs, the functionalized Ge surface ensures sufficient binding of protein and SNR to probe protein reactions at an atomic level and can be applied to both soluble and transmembrane proteins [37]. This work is particularly exciting because it does not require the use of SEIRAS, which shall be discussed below, to achieve spectra of monolayer levels of proteins.

A recent review by Rich and Iwaki highlights some of the key ATR-FTIR methods available to analyse protein transitions. The authors describe the experimental set ups required and advantages of combining ATR FTIR with photochemistry, electrochemistry and perfusion to explore redox reactions, ligand binding and conversions between catalytic intermediates [30]. The useful combination of photochemistry and ATR FTIR spectroscopy was also recently demonstrated by Bouhekka \& Bürgi [39]. In this study, the effect of visible light on $\mathrm{BSA}$ adsorbed onto $\mathrm{TiO}_{2}$ particles 
was examined in-situ using multiple reflections ATR FTIR spectroscopy. $\mathrm{TiO}_{2}$ is of interest because it is regularly used as biomedical implant material [40]. In situ measurements over time allowed structural transitions to be followed through the curve fitting of the second derivative spectra, as shown in Figure 3. It was shown that under visible light illumination the amide I band of adsorbed BSA shifted from $1653 \mathrm{~cm}^{-1}$ to $1648 \mathrm{~cm}^{-1}$ over a one hour time interval, indicating that the unordered structure within the protein had increased; this is highlighted by the red dashed line labelled Time in Figure 3 [39].

The study of protein behaviour using ATR FTIR spectroscopy is fundamentally limited by the signal to noise ratio (the intensity of the signal from the analyte should be 3 times stronger than that of noise to be considered detected and this criterion can be used to define the sensitivity of the system) that is achievable using current instruments. As such it is mostly limited to providing information on a macromolecular scale [41]. This does not negate the advantages such as the ease of sample preparation and the possibility for in-situ analysis, making it a highly valuable technique for protein conformational studies. It should be noted, however, that when combined with difference spectroscopy, it does allow for an increase in sensitivity comparable with SEIRAS, allowing molecular reactions to be followed [31,32], particularly when combined with chemically functionalized IREs [37].

\section{Surface Enhanced Infrared Absorption Spectroscopy}

Recent development in the area of Surface enhanced infrared absorption spectroscopy (SEIRAS) overcomes some of the limitations of sensitivity of detection presented by using traditional ATR FTIR spectroscopy to study proteins. Complimentary to surface enhanced Raman spectroscopy, this technique utilises the surface plasmon effect from the interaction of light with metallic nanoparticles $[42,43,44,45]$. These nanoparticles, mainly silver or gold are deposited onto the surface forming metal island films which provide localised enhancement of IR absorption. It is important to note that unlike SERS, the effect of enhancement in SEIRAS is not limited to gold or silver but has also been shown for other metals $[46,47]$. It is thought that the main contributing factor to the SEIRAS effect is the polarization of the metal island films by the incident IR radiation. This induced dipole generates a local electromagnetic field and the coupling of the local electromagnetic field with that of the incident IR radiation produces one that is stronger than the incident photon field, in the vicinity of the metal islands $[48,49]$. The dipolar moment of molecules absorbed onto the metal islands is enlarged because of this, enhancing the infrared absorption of the molecules. In addition the optical near field effect limits the enhancement to the vicinity of the metal island films, thus there is little contribution from the bulk sample to the measurement $[41,48,50]$. Chemical interactions also contribute to the SEIRAS effect [51]. The enhancement factor of the metal island films is highly dependent on the metal and on the morphology of the film [49,52]; those islands with greatest enhancement factors have been shown to be smaller in size than the incident radiation wavelength $[41,49]$. It should be noted, that one of the major drawbacks of SEIRAS can be the loss of spectral intensity due to the metal film deposited on the IRE surface if, for example, the film is too thick. Therefore, preparation of the metal island film is very important.

The signal enhancement of adsorbed protein achievable from SEIRAS is in the range of 2 orders of magnitude greater than without enhancement [50]. This technique was first applied to proteins by Ataka et al in 2003 [53] and further developments since have expanded its application to other 
protein studies $[41,43,50,54]$. The high sensitivity of the technique allows it to provide information from monolayer level and this is useful for examining the structure, function and assembly of molecules at the ATR interface. As with traditional ATR FTIR spectroscopy, measurements are carried out in-situ meaning that dynamic processes on a molecular level can be studied using SEIRAS [55]. Figure 4 shows the set-up for a typical SEIRAS experiment [43]. It is worth mentioning that the signal enhancement drops off rapidly within $10 \mathrm{~nm}$ from the surface and as such it is necessary to ensure that the biological material being studied is attached to the metal island film, often achieved through chemical modification of the film itself [43]. Polarization modulated infrared reflection spectroscopy (PMIRRAS) is also often used to study biological monolayers, particularly lipid monolayers $[56,57]$ Whilst this method can provide information on orientation and structure of protein monolayers, the technique lacks the sensitivity to detect minute structural changes that could occur during functional studies, which is one of the main advantages of SEIRAS [43].

Ataka's group is leading the field in applications of SEIRAS to proteins having pioneered its use to the study of membrane proteins in 2003 [53]. They have shown the potential of this method to study the catalytic action of membrane proteins driven by redox potential, in combination with electrochemistry [58]. The use of difference spectroscopy allows the changes in protein spectra to be observed following an induced perturbation. The SEIRAS arrangement can be easily adjusted to allow an electric current to be applied directly to the monolayer and from SEIRAS spectra; it is powerful in revealing the electron transfer mechanism of redox proteins.

SEIRAS has been applied increasingly to the study of membrane proteins because it has the potential to provide molecular level information on the structure, function and orientation of the membrane protein, often within the membrane itself $[43,54,59]$. Again by utilising difference spectroscopy, structural changes occurring in a self assembled monolayer can be monitored and it can also be used to study hydrogen bonding interactions in nucleic acids. Ataka et al were able to follow each step of the exchange of an oriented, immobilised monolayer of detergent solubilised membrane protein into a lipid bilayer via detergent substitution and then examine the protein funtionality, using SEIRAS [60]. This could potentially be applied to the study of the reaction mechanism of any membrane protein, particularly those which are medically and pharmaceutically important.

The development of biofunctional surfaces is a growing area because of their potential for use in medical devices where ensuring the proper orientation and functionality of the protein is critical. However, one of the major challenges within the design of the surfaces is the ability to assess the functionality of the surface $[41,50]$. SEIRAS has the ability to provide molecular information on proteins in monolayers and can be performed in-situ preserving the integrity of the monolayers. The orientation of proteins within the monolayer can be also determined from the infrared spectra [54].

One of the major advances in the use of SEIRAS is within the field of biosensing and immunoassays to provide information on the binding mechanisms underlying biological function $[41,61,62]$. The use of gold nanoparticles can allow for the competitive adsorption of different molecules to be examined [63]. Following the formation of the gold film, a monolayer of selected antibody or antigen is deposited to form a biosensing layer. Xu et al. used anti rabbit antibodies (anti rlgG) from goat to study the binding mechanism of the corresponding antigen [61]. A background measurement is taken after the formation of the biosensing layer meaning only molecules that specifically bind to 
the substrate can be detected in future measurements. SEIRAS successfully allowed the target rlgG bound onto the surface to be observed by molecular recognition (Figure 5), allowing in-situ monitoring of the immunoreactions [61]. From the absorption data gathered it is possible to determine the reaction kinetics of the antigen-antibody binding. All of which can be achieved in-situ without the need for additional dyes or labels and as such this method shows considerable promise for real time sensing of biomolecular bonding [61].

Gold nanoparticles have a number of uses within biomedical applications and in combination with ATR FTIR spectroscopy. In some cases, nanoparticles are used to introduce drugs into specific sites because they have a particularly good uptake within cells [64]. Another exciting application presented by Lane and Seo demonstrated that ATR FTIR spectroscopy can be used to distinguish between cancerous and benign cells containing gold nanoparticles [64]. The presence of the nanoparticles within the cells allows an enhanced IR absorbance, similar to the effect seen when the surface is modified as described above. The study by Lane and Seo [64] shows the increase in spectral intensity with increasing gold nanoparticles concentration for cancerous and normal cell lines, which could allow for any spectral differences to be seen more easily. This development further emphasises the potential of ATR FTIR spectroscopy for medical diagnostics and is not limited by the reproducibility issues still present within SEIRAS studies. This approach does not depend on the formation of metal island films, like in SEIRAS approach, as the gold nanoparticles are present within the cells themselves and the level of signal enhancement is dependent on the concentration of nanoparticles[64]. It is also possible to study cells using ATR FTIR without the use of gold nanoparticles, as shall be discussed below. However, the demonstration of the enhanced IR absorbance achieved when the nanoparticles are present could have some interesting applications, particularly to study the delivery of drugs within cells.

Despite these exciting advances and the growing use of SEIRAS in protein studies, there is still potential for further development. This mainly concerns the development of a reproducible method for surface preparation and in turn, a greater understanding of the mechanism of SEIRAS [41]. Once this has been achieved, SEIRAS could have a much greater impact within proteomics studies and could be used more reliably within medical diagnostics. At present there is still too much uncertainty for it to be used routinely in these areas [61,63].

\section{ATR FTIR spectroscopic imaging}

Spectroscopic imaging couples an FTIR spectrometer with an infrared array detector to allow for the simultaneous collection of the infrared spectra of a sample from multiple locations within the sample. This allows the generation of chemical images indicating locations of high absorbance at a particular wavenumber for different components within a sample. Whereas, previously these chemical images were generated by time consuming point by point mapping of a sample, the development of infrared array detectors has rapidly improved the ability to obtain infrared images. Linear array detectors allow for an image to be obtained through rastering of rows of a sample in comparison with focal plane array detectors that collect the infrared absorbance from all locations within a sample simultaneously. Both approaches have advantages and disadvantages which are discussed at length elsewhere $[13,65,66]$. The main advantage of using an FPA detector is that since all the data are acquired simultaneously it is particularly suitable for the study of dynamic systems and multiple samples; this is not the case with the linear array detector $[65,67]$. It should be noted, 
however, that linear array detectors have better signal to noise ratios and as such may be more suitable for sensitive analysis [65]. In both instances, it is possible to obtain detailed chemical and spatial information from samples and the variety of sampling arrangements available means that this chemical imaging approach has opened up some exciting opportunities for protein studies.

Macro ATR FTIR spectroscopic imaging is performed without use of a microscope and instead it couples an FTIR spectrometer, equipped with an inverted prism ATR accessory, with a focal plane array detector. The detector size can be varied between $64 \times 64$ pixels up to $128 \times 128$ pixels allowing between 4096 to 16384 spectra to be obtained from the sample in one measurement. This imaging approach can be used with different ATR accessories such as the imaging diamond Golden Gate accessory (Specac) or a silicon inverted prism ATR accessory (Pike), which allows for a range of different imaging areas and spatial resolutions. There are dedicated reviews discussing this, the most recent by Kazarian and Chan is very comprehensive $[13,65,68]$.

ATR-FTIR spectroscopic imaging in its macro and micro modes has been applied across many different areas of research, from pharmaceutical tablet analysis [69,70], art conservation [67] through to understanding the fouling in heat exchangers [71]. It has also been applied in several biomedical studies such as to the study of arthrosclerosis plaques in arteries [72] and drug permeation through skin [73]. The most relevant applications, which shall be highlighted here, focus on the inherent capacity of this approach for high throughput (HTP) studies [74,75]. High throughput studies allow for the analysis of multiple samples simultaneously. This can save both time and money by reducing the amount of sample required and also the time spent on analysis, especially in optimisation trials. In the case of macro ATR-FTIR spectroscopic imaging, high throughput studies have been carried using either a PDMS or wax grids defining multiple areas for sample deposition on the surface of an IRE used. The wax grid can be printed directly onto the measuring surface of an IRE (ATR crystal) using a microdroplet system. This system can also be used for the deposition of nanolitre volumes of samples onto the surface.

This approach was applied to the crystallisation of proteins as a means of optimising for crystallisation conditions [76]. Protein crystallisation is seen as an important step in structural proteomics studies [77] and the use of various high throughput approaches to optimise this step have had major impact on the field [78]. A common method of protein crystallisation is microbatch crystallisation where the crystallisation occurs in aqueous medium placed under oil. This method can be used directly on the surface of an inverted prism as shown in Figure 6, where PDMS wells are used to contain the oil. A protein and precipitant sample can be deposited prior to (as in a) or after the oil is in place (as in b), since the protein/precipitant solution is less dense than the oil, the drop of the solution will then displace oil on the surface of the ATR prism allowing it and not the oil to be measured by ATR-FTIR imaging. For HTP studies, a wax grid was printed on the surface allowing up to six different crystallisation conditions to be studied simultaneously. In this case the second method of deposition was used as it prevents evaporation from the solution drops whilst the deposition takes place, although it does reduce the degree of control one has for positioning the samples [76]. Figure 7a shows the crystallisation of Lysozyme on a ZnSe ATR crystal where the location of crystals is determined from the plotting the distribution of the integrated amide II band. When the optical image in Figure $7 b$ is overlaid with the infrared image, as in Figure $7 c$, it can be seen that the locations of the crystals in the ATR image correspond almost entirely with those in the optical image, confirming that this technique is successfully identifying protein crystals. The use of 
macro ATR FTIR imaging to optimise for protein crystallisation conditions is advantageous as it allows one to determine if crystals formed are indeed protein rather than salt crystals without moving or destroying the crystals, a common problem in crystallisation studies [76]. Possibilities exist to expand this to study to studies of many more samples simultaneously and to proteins that are more challenging to crystallise and study.

Continuing along the high throughput analysis strategy, another exciting application of macro ATR FTIR imaging combining the use of surface modification and protein studies [79]. In this work, the measuring surface of a silicon ATR crystal was modified using standard self assembled monolayer preparation techniques to create a gradient of properties along the surface of the crystal as shown in Figure 8. A gradient of hydrophobicity was created using a modified vapour diffusions approach where a reservoir containing Octyltrichlorosilane (OTS) solution was allowed to evaporate over a silicon ATR surface that had previously been cleaned with piranha solution to expose the hydroxyl groups. As such, areas closest to the reservoir were exposed to a higher concentration of OTS vapour and hence were more hydrophobic than areas further away. This development allows imaging with an FPA detector for sensitive surface detection, something that was previously thought to be too challenging due to signal noise limiting factors [79]. The authors overcame this SNR issue through the use of pixel averaging along the horizontal axis of the ATR image. By averaging each row of pixels, the gradient of monolayer along the surface was measured without degrading the spatial resolution along the vertical axis. Given the interest in the effect of surfaces on protein adsorption, as discussed early, the gradient surface was then used to examine Lysozyme adsorption and crystallisation on the surface of the silicon ATR crystal. It was shown that as the hydrophobicity decreased along the surface, the amount of protein adsorbed decreased as did the number of crystals formed, Figure 9 [79]. This application is the first step in realising the study of protein structure and function using ATR FTIR imaging in combination with high throughput surface properties. However, it should be noted that currently the sensitivity of the system is such that the adsorbed protein spectra are too noisy for reliable secondary structural analysis.

Recent advances in the area have also seen the combination of macro imaging and microfluidics $[80,81]$. Using a computerised wax printing device, microfluidic channels can be printed either directly onto the surface of an ATR element or onto transmission slides. This has been applied to the study of reactions [80] and cells, however as interest in protein crystallisation using novel methods such as microfluidics continues to be explored $[82,83]$, this method could easily be adapted for this and given the advantages of allowing proteinaceous crystals to be identified in situ without additional labels, this presents a future application for the field. Using transmission FTIR, work has been carried out to study protein conformational changes due to $\mathrm{pH}$ in microfluidic flows [84], there is potential for this to also be applied using FTIR imaging. It should also be noted that studies of live cells in microfluidic devices in situ using FTIR spectroscopic imaging in transmission mode has also became possible very recently due to new developments in methodology [85].

Micro ATR FTIR imaging offers opportunities for protein studies. In this arrangement, an infrared microscope objective is combined with a Cassegrain objective allowing spatial resolutions of up to 4 $\mu \mathrm{m}$ to be realised [13]. The high spatial resolution of micro ATR imaging means that it is particularly advantageous to the study of biological materials such as tissues, cells and arteries [72,86]. Most recently, this has been used to study hanging drop protein crystallisation [87]. A drop of protein and precipitant solution are deposited onto a removable Germanium ATR objective and suspended 
above a reservoir in exactly the same manner as conventional hanging drop crystallisation studies. This can be seen in the schematic of Figure 10. By measuring the growth of proteins in-situ, it is possible to detect the early stages of crystals grown and the formation of micro protein crystals, confirming the crystals are in fact protein rather than salt [87]. There may also by additional insight into the growth kinetic of protein crystals through analysis of secondary structure from the IR spectrum. However, one of the drawbacks of this approach is the limited imaging area. At only 64 $\mu \mathrm{m} \times 64 \mu \mathrm{m}$ [13], there is no way to guarantee that crystals will grow within this area. There could be potential to overcome this using seeding or surface modifications [87].

A recent review by Miller et al discussed the application of FTIR microspectroscopic imaging to the study of protein aggregation within cells [88]. The method discussed in the review mostly concentrates on operation in transmission mode and using a synchrotron source. Whilst the use of synchrotron source can allow higher SNR to be achieved and can allow sub-cellular information to be obtained from a sample [89], it is still currently limited to use with transmission mode, which, as previously discussed, requires a very short path length for aqueous samples. Chan et al. showed that micro ATR imaging can provide high spatial resolution without the need for synchrotron source [90]. The spatial resolution achievable with micro ATR FTIR imaging is dependent on the numerical aperture of the system and wavelength of light which, when combined with a germanium ATR crystal of high refractive index means that high spatial resolutions can be reached, in comparison with transmission that uses objectives with lower numerical apertures (e.g. four times lower) $[13,90]$ Micro ATR FTIR imaging has previously been demonstrated allowing the measurement of live and fixed cells without the need for synchrotron source $[65,91]$.

\section{Future perspectives}

This review has highlighted the potential of ATR FTIR spectroscopy for the study of proteins, particularly focusing on recent applications of ATR FTIR imaging and SEIRAS. Further development within these areas will enhance the applicability of this powerful analytical tool for studies of proteins. The limitations of SEIRAS in terms of reproducibility of substrates and the signal to noise limitations of ATR FTIR imaging spectra for analysing secondary structure could well be overcome within the next several years. Preliminary analysis using a combination of SEIRAS and ATR FTIR imaging shows that this approach is both feasible [13] and could potentially allow for more in-depth study of the effect of chemistry and topography of surfaces on protein conformation, in a high throughput manner. There is much potential for its use in other areas of protein research such as the purification and separation of proteins as well as biopharmaceutical development. Also inevitably, as more work is carried out within the field, the ease of secondary structure characterisation will increase along with the information that can be gained from IR spectra. However long these developments take, the main advantages of ATR FTIR spectroscopy will still remain in that it is a powerful, label free and non destructive technique that can be used to provide sensitive in-situ analysis of proteins across a wide range of systems.

\section{References}

[1] J.M. Chandonia, S.E. Brenner, The impact of structural genomics: Expectations and outcomes, Science 311 (2006) 347-351. 
[2] V. Bolanos Garcia, N. Chayen, N. Chayen, New directions in conventional methods of protein crystallization, Progress in biophysics and molecular biology 101 (2009) 3-12.

[3] V.N. Uversky, Natively unfolded proteins: A point where biology waits for physics, Protein Science 11 (2002) 739-756.

[4] R.M. Bill, P.J.F. Henderson, S. Iwata, E.R.S. Kunji, H. Michel, R. Neutze, S. Newstead, B. Poolman, C.G. Tate, H. Vogel, Overcoming barriers to membrane protein structure determination, Nature Biotechnology 29 (2011) 335-340.

[5] D.A.P. Gutmann, E. Mizohata, S. Newstead, S. Ferrandon, V. Postis, X.B. Xia, P.J.F. Henderson, H.W. Van Veen, B. Byrne, A high-throughput method for membrane protein solubility screening: The ultracentrifugation dispersity sedimentation assay (vol 16, pg 1422, 2007), Protein Science 16 (2007) 2775-2775.

[6] A.A.H. Shukla, P.; Gupta, P.; Yigzaw, Y.; Hubbard, B, Strategies to address aggregation during Protein A chromatography., Bioprocess International 3 (2005) 36 - 45

[7] S. Gerontas, M.S. Shapiro, D.G. Bracewell, Chromatography modelling to describe protein adsorption at bead level, Journal of Chromatography A 1284 (2013) 44-52.

[8] S. Matheus, W. Friess, H.C. Mahler, FTIR and nDSC as analytical tools for high-concentration protein formulations, Pharmaceutical Research 23 (2006) 1350-1363.

[9] Z.W. Ma, Z.W. Mao, C.Y. Gao, Surface modification and property analysis of biomedical polymers used for tissue engineering, Colloids and Surfaces B-Biointerfaces 60 (2007) 137-157.

[10] K. Nakanishi, T. Sakiyama, K. Imamura, On the adsorption of proteins on solid surfaces, a common but very complicated phenomenon, Journal of Bioscience and Bioengineering 91 (2001) 233-244.

[11] K.K. Chittur, FTIR/ATR for protein adsorption to biomaterial surfaces, Biomaterials 19 (1998) 357-369.

[12] Y. Mei, T. Wu, C. Xu, K.J. Langenbach, J.T. Elliott, B.D. Vogt, K.L. Beers, E.J. Amis, N.R. Washburn, Tuning cell adhesion on gradient poly(2-hydroxyethyl methacrylate)-grafted surfaces, Langmuir 21 (2005) 12309-12314.

[13] S.G. Kazarian, K.L.A. Chan, Micro- and Macro-Attenuated Total Reflection Fourier Transform Infrared Spectroscopic Imaging, Applied Spectroscopy 64 (2010) 135A-152A.

[14] S. Krimm, Infrared spectra and chain conformation of proteins, Journal of Molecular Biology 4 (1962) 528-540.

[15] D.M. Byler, H. Susi, Examination of the secondary structure of proteins by deconvolved FTIR spectra, Biopolymers 25 (1986) 469-487.

[16] T. Miyazawa, E.R. Blout, The Infrared Spectra of Polypeptides in Various Conformations: Amide I and II Bands1, Journal of the American Chemical Society 83 (1961) 712-719.

[17] P.I. Haris, F. Severcan, FTIR spectroscopic characterization of protein structure in aqueous and non-aqueous media, Journal of Molecular Catalysis B-Enzymatic 7 (1999) 207-221.

[18] A. Barth, C. Zscherp, What vibrations tell us about proteins, Quarterly reviews of biophysics 35 (2002) 369-430.

[19] R.W. Sarver Jr, W.C. Krueger, Protein secondary structure from fourier transform infrared spectroscopy: A data base analysis, Analytical Biochemistry 194 (1991) 89-100.

[20] W.K. Surewicz, H.H. Mantsch, D. Chapman, Determination of protein secondary structure by Fourier-Transform Infrared spectroscopy - A critical assessment Biochemistry 32 (1993) 389394.

[21] V.A. Shashilov, I.K. Lednev, Advanced Statistical and Numerical Methods for Spectroscopic Characterization of Protein Structural Evolution, Chemical Reviews 110 (2010) 5692-5713.

[22] J. Trevisan, P.P. Angelov, P.L. Carmichael, A.D. Scott, F.L. Martin, Extracting biological information with computational analysis of Fourier-transform infrared (FTIR) biospectroscopy datasets: current practices to future perspectives, Analyst 137 (2012) 32023215. 
[23] E. Goormaghtigh, J.M. Ruysschaert, V. Raussens, Evaluation of the information content in infrared spectra for protein secondary structure determination, Biophysical Journal 90 (2006) 2946-2957.

[24] A. Dong, P. Huang, W.S. Caughey, Protein secondary structures in water from 2nd-derivative Amide I Infrared spectra, Biochemistry 29 (1990) 3303-3308.

[25] J. Kong, S. Yu, Fourier transform infrared spectroscopic analysis of protein secondary structures, Acta Biochimica Et Biophysica Sinica 39 (2007) 549-559.

[26] P. Cha, A. Krishnan, V.F. Fiore, E.A. Vogler, Interfacial energetics of protein adsorption from aqueous buffer to surface with varying hydrophilicity, Langmuir 24 (2008) 2553-2563.

[27] A. Sethuraman, G. Belfort, Protein structural perturbation and aggregation on homogeneous surfaces, Biophysical Journal 88 (2005) 1322-1333.

[28] R.J. Green, I. Hopkinson, R.A.L. Jones, Unfolding and intermolecular association in globular proteins adsorbed at interfaces, Langmuir 15 (1999) 5102-5110.

[29] P.M. Reynolds, R.H. Pedersen, J. Stormonth-Darling, M.J. Dalby, M.O. Riehle, N. Gadegaard, Label-Free Segmentation of Co-cultured Cells on a Nanotopographical Gradient, Nano Letters 13 (2013) 570-576.

[30] P.R. Rich, M. Iwaki, Methods to probe protein transitions with ATR infrared spectroscopy, Molecular Biosystems 3 (2007) 398-407.

[31] P. Pinkerneil, J. Guldenhaupt, K. Gerwert, C. Kotting, Surface-Attached Polyhistidine-Tag Proteins Characterized by FTIR Difference Spectroscopy, Chemphyschem 13 (2012) 26492653.

[32] P. Rigler, W.P. Ulrich, P. Hoffmann, M. Mayer, H. Vogel, Reversible immobilization of peptides: Surface modification and in situ detection by attenuated total reflection FTIR Spectroscopy, Chemphyschem 4 (2003) 268-275.

[33] D.S. Salloum, J.B. Schlenoff, Protein adsorption modalities on polyelectrolyte multilayers, Biomacromolecules 5 (2004) 1089-1096.

[34] J.C.V. P. Schwinté, C. Picart, Y. Haikel, P. Schaaf, B. Szalontai, Stabilizing Effects of Various Polyelectrolyte Multilayer Films on the Structure of Adsorbed/Embedded Fibrinogen Molecules: An ATR-FTIR Study+, The journal of physical chemistry. B 105 (2001) 1190611916.

[35] G. Steiner, S. Tunc, M. Maitz, R. Salzer, Conformational Changes during Protein Adsorption. FTIR Spectroscopic Imaging of Adsorbed Fibrinogen Layers, Analytical Chemistry 79 (2007) 1311-1316.

[36] K. Onodera, A. Hirano-Iwata, K.I. Miyamoto, Y. Kimura, M. Kataoka, Y. Shinohara, M. Niwano, Label-free detection of protein-protein interactions at the GaAs/Water interface through surface infrared spectroscopy: Discrimination between specific and nonspecific interactions by using secondary structure analysis, Langmuir 23 (2007) 12287-12292.

[37] J. Schartner, J. Guldenhaupt, B. Mei, M. Rogner, M. Muhler, K. Gerwert, C. Kotting, Universal Method for Protein Immobilization on Chemically Functionalized Germanium Investigated by ATR-FTIR Difference Spectroscopy, Journal of the American Chemical Society 135 (2013) 4079-4087.

[38] A. Aamouche, E. Goormaghtigh, FTIR-ATR biosensor based on self-assembled phospholipids surface: Haemophilia factor VIII diagnosis, Spectroscopy-an International Journal 22 (2008) 223-234.

[39] A. Bouhekka, T. Burgi, In situ ATR-IR spectroscopy study of adsorbed protein: Visible light denaturation of bovine serum albumin on TiO2, Applied Surface Science 261 (2012) 369-374.

[40] I. Dolamic, T. Burgi, In Situ ATR-IR Study on the Photocatalytic Decomposition of Amino Acids over Au/TiO2 and TiO2, Journal of Physical Chemistry C 115 (2011) 2228-2234.

[41] L.-X. Wang, X.-E. Jiang, Bioanalytical Applications of Surface-enhanced Infrared Absorption Spectroscopy, Chinese Journal of Analytical Chemistry 40 (2012) 975-982. 
[42] R.F. Aroca, D.J. Ross, C. Domingo, Surface-enhanced infrared spectroscopy, Applied Spectroscopy 58 (2004) 324A-338A.

[43] K. Ataka, T. Kottke, J. Heberle, Thinner, Smaller, Faster: IR Techniques To Probe the Functionality of Biological and Biomimetic Systems, Angewandte Chemie-International Edition 49 (2010) 5416-5424.

[44] M. Osawa, M. Kuramitsu, A. Hatta, W. Suetaka, Electricmagnetic effect in enhanced infraredabsorption of adsorbed molecules on thin metal-films, Surface Science 175 (1986) L787L793.

[45] M. Osawa, M. Ikeda, Surface enhanced infrared-absorption of para-nitrobenzoic acid deposited on silver island films - contributions of eletromagnetic and chemical mechanisms Journal of Physical Chemistry 95 (1991) 9914-9919.

[46] D.A. Heaps, P.R. Griffiths, Band shapes in the infrared spectra of thin organic films on metal nanoparticles, Vibrational Spectroscopy 42 (2006) 45-50.

[47] A. Hartstein, J.R. Kirtley, J.C. Tsang, Enhancement of the infrared-absorption from molecular monolayers with thin metal overlayers, Physical Review Letters 45 (1980) 201-204.

[48] M. Osawa, K. Ataka, K. Yoshii, Y. Nishikawa, Surface-enhanced infrared spectroscopy - The origin of the absorption enhancement and band selection rule in the infrared-spectra of molecules adsorbed on fine metal particles Applied Spectroscopy 47 (1993) 1497-1502.

[49] Y. Nishikawa, T. Nagasawa, K. Fujiwara, M. Osawa, Silver island films for surface-enhanced infrared-absorption spectroscopy - Effect of island morphology on the absorption enhancement. , Vibrational Spectroscopy 6 (1993) 43-53.

[50] K. Ataka, J. Heberle, Biochemical applications of surface-enhanced infrared absorption spectroscopy, Analytical and Bioanalytical Chemistry 388 (2007) 47-54.

[51] G.T. Merklin, P.R. Griffiths, Influence of chemical interactions on the surface-enhanced infrared absorption spectrometry of nitrophenols on copper and silver films, Langmuir 13 (1997) 6159-6163.

[52] G.T. Merklin, P.R. Griffiths, Effect of microscopic surface roughness in surface-enhanced infrared absorption spectrometry, Journal of Physical Chemistry B 101 (1997) 5810-5813.

[53] K. Ataka, J. Heberle, Electrochemically induced surface-enhanced infrared difference absorption (SEIDA) spectroscopy of a protein monolayer, Journal of the American Chemical Society 125 (2003) 4986-4987.

[54] X. Jiang, A. Zuber, J. Heberle, K. Ataka, In situ monitoring of the orientated assembly of streptagged membrane proteins on the gold surface by surface enhanced infrared absorption spectroscopy, Physical Chemistry Chemical Physics 10 (2008) 6381-6387.

[55] A. Hirano-Iwata, R. Yamaguchi, K. Miyamoto, Y. Kimura, M. Niwano, In situ real-time monitoring of biomolecular interactions by using surface infrared spectroscopy, Journal of Applied Physics 105 (2009).

[56] I. Cornut, B. Desbat, J.M. Turiet, J. Dufourcq, In situ study by polarization modulated Fourier transform infrared spectroscopy of the structure and orientation of lipids and amphipathic peptides at the air-water interface, Biophysical Journal 70 (1996) 305-312.

[57] J.C.P. Damalio, T.M. Nobre, J.L. Lopes, O.N. Oliveira, A.P.U. Araujo, Lipid interaction triggering Septin2 to assembly into beta-sheet structures investigated by Langmuir monolayers and PM-IRRAS, Biochimica Et Biophysica Acta-Biomembranes 1828 (2013) 1441-1448.

[58] K. Ataka, J. Heberle, Bioenergetics at the gold surface: SEIRAS probes photosynthetic and respiratory reactions at the monolayer level, Biochemical Society Transactions 36 (2008) 986-991.

[59] K. Ataka, J. Heberle, Use of surface enhanced infrared absorption spectroscopy (SEIRA) to probe the functionality of a protein monolayer, Biopolymers 82 (2006) 415-419.

[60] K. Ataka, F. Giess, W. Knoll, R. Naumann, S. Haber-Pohlmeier, B. Richter, J. Heberle, Oriented attachment and membrane reconstitution of his-tagged cytochrome c oxidase to a gold 
electrode: In situ monitoring by surface-enhanced infrared absorption spectroscopy, Journal of the American Chemical Society 126 (2004) 16199-16206.

[61] J. Xu, T. Chen, W. Bao, K. Wang, X. Xia, Label-Free Strategy for In-Situ Analysis of Protein Binding Interaction Based on Attenuated Total Reflection Surface Enhanced Infrared Absorption Spectroscopy (ATR-SEIRAS), Langmuir 28 (2012) 17564-17570.

[62] C.W. Brown, Y. Li, J.A. Seelenbinder, P. Pivarnik, A.G. Rand, S.V. Letcher, O.J. Gregory, M.J. Platek, Immunoassays based on surface enhanced infrared absorption spectroscopy, Analytical Chemistry 70 (1998) 2991-2996.

[63] D.H. Tsai, M. Davila-Morris, F.W. DelRio, S. Guha, M.R. Zachariah, V.A. Hackley, Quantitative Determination of Competitive Molecular Adsorption on Gold Nanoparticles Using Attenuated Total Reflectance-Fourier Transform Infrared Spectroscopy, Langmuir 27 (2011) 9302-9313.

[64] R. Lane, S.S. Seo, Attenuated Total Reflectance Fourier Transform Infrared Spectroscopy Method to Differentiate Between Normal and Cancerous Breast Cells, Journal of Nanoscience and Nanotechnology 12 (2012) 7395-7400.

[65] S.G. Kazarian, K.L.A. Chan, ATR-FTIR spectroscopic imaging: recent advances and applications to biological systems, Analyst 138 (2013) 1940-1951.

[66] G. Steiner, E. Koch, Trends in Fourier transform infrared spectroscopic imaging, Analytical and Bioanalytical Chemistry 394 (2009) 671-678.

[67] E. Joseph, C. Ricci, S.G. Kazarian, R. Mazzeo, S. Prati, M. loele, Macro-ATR-FT-IR spectroscopic imaging analysis of paint cross-sections, Vibrational Spectroscopy 53 (2010) 274-278.

[68] S.G. Kazarian, K.L.A. Chan, Applications of ATR-FTIR spectroscopic imaging to biomedical samples, Biochimica Et Biophysica Acta-Biomembranes 1758 (2006) 858-867.

[69] P. Wray, K.L.A. Chan, J. Kimber, S.G. Kazarian, Compaction of pharmaceutical tablets with different polymer matrices studied by FTIR imaging and X-ray microtomography, Journal of Pharmaceutical Sciences 97 (2008) 4269-4277.

[70] J.A. Kimber, S.G. Kazarian, F. Stepanek, Microstructure-based mathematical modelling and spectroscopic imaging of tablet dissolution, Computers \& Chemical Engineering 35 (2011) 1328-1339.

[71] F.H. Tay, S.G. Kazarian, Study of Petroleum Heat-exchanger Deposits with ATR-FTIR Spectroscopic Imaging, Energy \& Fuels 23 (2009) 4059-4067.

[72] F. Palombo, S.G. Cremers, P.D. Weinberg, S.G. Kazarian, Application of Fourier transform infrared spectroscopic imaging to the study of effects of age and dietary L-arginine on aortic lesion composition in cholesterol-fed rabbits, Journal of the Royal Society Interface 6 (2009) 669-680.

[73] J.M. Andanson, K.L.A. Chan, S.G. Kazarian, High-Throughput Spectroscopic Imaging Applied to Permeation Through the Skin, Applied Spectroscopy 63 (2009) 512-517.

[74] K.L.A. Chan, S.G. Kazarian, D. Vassou, V. Gionis, G.D. Chryssikos, In situ high-throughput study of drug polymorphism under controlled temperature and humidity using FT-IR spectroscopic imaging, Vibrational Spectroscopy 43 (2007) 221-226.

[75] K.L.A. Chan, S.G. Kazarian, Fourier transform infrared imaging for high-throughput analysis of pharmaceutical formulations, Journal of Combinatorial Chemistry 7 (2005) 185-189.

[76] K.L.A. Chan, L. Govada, R.M. Bill, N.E. Chayen, S.G. Kazarian, Attenuated Total Reflection-FT-IR Spectroscopic Imaging of Protein Crystallization, Analytical Chemistry 81 (2009) 3769-3775.

[77] A. McPherson, Introduction to protein crystallization, Methods 34 (2004) 254-265.

[78] E. Saridakis, Perspectives on High-Throughput Technologies Applied to Protein Crystallization, Protein and Peptide Letters 19 (2012) 778-783.

[79] S. Glassford, K.L.A. Chan, B. Byrne, S.G. Kazarian, Chemical Imaging of Protein Adsorption and Crystallization on a Wettability Gradient Surface, Langmuir 28 (2012) 3174-3179.

[80] K.L.A. Chan, S.G. Kazarian, FT-IR Spectroscopic Imaging of Reactions in Multiphase Flow in Microfluidic Channels, Analytical Chemistry 84 (2012) 4052-4056. 
[81] K.L.A. Chan, X.Z. Niu, A.J. de Mello, S.G. Kazarian, Rapid prototyping of microfluidic devices for integrating with FT-IR spectroscopic imaging, Lab on a Chip 10 (2010) 2170-2174.

[82] Y. Yu, X. Wang, D. Oberthur, A. Meyer, M. Perbandt, L. Duan, Q. Kang, Design and application of a microfluidic device for protein crystallization using an evaporation-based crystallization technique, Journal of Applied Crystallography 45 (2012) 53-60.

[83] L. Li, R.F. Ismagilov, Protein Crystallization Using Microfluidic Technologies Based on Valves, Droplets and SlipChip, in: D.C. Rees, K.A. Dill, J.R. Williamson (Eds.), Annual Review of Biophysics, Vol 39, Annual Reviews, Palo Alto, 2010, pp. 139-158.

[84] D. Prim, S. Crelier, J.M. Segura, Coupling of a Microfluidic Mixer to a Fourier-transform Infrared Spectrometer for Protein-Conformation Studies, Chimia 65 (2011) 815-816.

[85] K.L.A. Chan, S.G. Kazarian, Aberration-free FTIR spectroscopic imaging of live cells in microfluidic devices, Analyst 138 (2013) 4040-4047.

[86] M.K. Kuimova, K.L.A. Chan, S.G. Kazarian, Chemical Imaging of Live Cancer Cells in the Natural Aqueous Environment, Applied Spectroscopy 63 (2009) 164-171.

[87] S.E. Glassford, L. Govada, N.E. Chayen, B. Byrne, S.G. Kazarian, Micro ATR FTIR imaging of hanging drop protein crystallisation, Vibrational Spectroscopy 63 (2012) 492-498.

[88] L.M. Miller, M.W. Bourassa, R.J. Smith, FTIR spectroscopic imaging of protein aggregation in living cells, Biochimica et Biophysica Acta (BBA) - Biomembranes (2013).

[89] M.J. Nasse, M.J. Walsh, E.C. Mattson, R. Reininger, A. Kajdacsy-Balla, V. Macias, R. Bhargava, C.J. Hirschmugl, High-resolution Fourier-transform infrared chemical imaging with multiple synchrotron beams, Nature Methods 8 (2011) 413-U458.

[90] K.L.A. Chan, S.G. Kazarian, A. Mavraki, D.R. Williams, Fourier transform infrared imaging of human hair with a high spatial resolution without the use of a synchrotron, Applied Spectroscopy 59 (2005) 149-155.

[91] T.P. Wrobel, K.M. Marzec, K. Majzner, K. Kochan, M. Bartus, S. Chlopicki, M. Baranska, Attenuated total reflection Fourier transform infrared (ATR-FTIR) spectroscopy of a single endothelial cell, Analyst 137 (2012) 4135-4139.

\section{Figure Captions}

Figure 1 - FTIR spectrum of a typical protein illustrating the Amide I and Amide II bands at $\sim 1650 \mathrm{~cm}$ 1 and $\sim 1540 \mathrm{~cm}-1$, respectively. (Inset) Expanded view of the Amide I band, which can be deconvolved into its secondary structure component (Reprinted from R.W. Sarver and W.C. Krueger, Protein secondary structure from fourier transform infrared spectroscopy: A data base analysis, Analytical Biochemistry 194 (1991) 89-100, with permission from Elsevier). [19]

Figure 2 - Evanescent wave electric field amplitude (E) falls off exponentially away from the surface of the crystal. (A) BSA adsorption on the negatively charged surface. (B) BSA adsorption on the positively charged surface. $d p$ represents the penetration depth of the evanescent wave normal to the surface of the crystal, using Ge ( $n$ ) 4.0) in contact with the medium of n ) 1.42. (Reprinted with permission from D.S. Salloum and J.B. Schlenoff, Protein Adsorption Modalites on Polyelectrolyte Multilayers, Biomacromolecules, 5 (2004) 1089-1096. Copyright (2004) American Chemical Society). [33]

Figure 3 - Second derivative of amide I region for adsorption spectrum of BSA on TiO2 under rinsing by water and irradiation with visible light (VL) for around $1 \mathrm{~h}$. Intervals between spectra are around $10 \mathrm{~min}$ and the black dashed line labelled time shows indicates the order of spectral measurements (Reprinted from A. Bouhekka and T. Bürgi, In situ ATR-IR spectroscopy study of adsorbed protein: 
Visible light denaturation of bovine serum albumin on $\mathrm{TiO}_{2}$, Applied Surface Science 261 (2012) 369374, with permission from Elsevier). [39]

Figure 4 - Experimental set up for SEIRAS. (Reprinted with permission from K. Ataka, T. Kottke and J. Heberle, Thinner, Smaller, Faster: IR Techniques To Probe the Functionality of Biological and Biomimetic System, Angewandte Chemie-International Edition, 49 (2010) 5416 - 5424. Copyright (2010) John Wiley and Sons). [43]

Figure 5 - (a) Background spectrum of the goat anti-rlgG modified substrate in PBS. (b) SEIRA spectrum of chicken IgG $(0.3 \mu \mathrm{g} / \mathrm{mL})$. (c) Hemoglobin $(3 \mathrm{mg} / \mathrm{mL})$, and (d) $\mathrm{rlgG}(0.3 \mu \mathrm{g} / \mathrm{mL})$ after 35 min immunobinding to goat anti-rlgG surface. Before each injection of protein solution, the chamber was rinsed with pure PBS buffer (Reprinted with permission from J. Xu, T. Chen, W. Bao, K. Wang and X. Xia, Label-Free Strategy for In-Situ Analysis of Protein Binding Interaction Based on Attenuated Total Reflection Surface Enhanced Infrared Spectroscopy (ATR-SEIRAS), Langmuir, 28 (2012) 1756417570. Copyright (2012) American Chemical Society). [61]

Figure 6 - Schematic diagram showing different strategies for sample deposition. (Reprinted with permission from K.L. A. Chan, L. Govada, R. Bill, N.E. Chayen and S.G. Kazarian, Attenuated Total reflection-FT-IR Spectroscopic imaging of Protein Crystallization, Analytical Chemistry, 81 (2009) 3769-3775. Copyright (2009) American Chemical Society). [76]

Figure 7 - (a) ATR-FT-IR image (imaged area is approximately $2.5 \times 3.6 \mathrm{~mm} 2$ ) of the protein crystal formed on the measuring surface $20 \mathrm{~h}$ after the introduction of the crystallizing agent. Red represents high protein concentration. The visible image (b) shows the protein crystals formed coincide well with the location of the red spots on the ATR-FT-IR image. (c) Result of overlaying images (a) and (b). Five out of 50 crystals (highlighted with arrows) were not captured in the ATR-FTIR image and 3 crystals (circled) were mislocated. (Reprinted with permission from K.L. A. Chan, L. Govada, R. Bill, N.E. Chayen and S.G. Kazarian, Attenuated Total reflection-FT-IR Spectroscopic imaging of Protein Crystallization, Analytical Chemistry, 81 (2009) 3769-3775. Copyright (2009) American Chemical Society). [76]

Figure 8 - Schematic diagram of method for preparation of gradient of OTS on silicon ATR element. (Reprinted with permission from S. Glassford, K.L.A. Chan, B. Byrne and S.G. Kazarian, Chemical Imaging of Protein Adsorption and Crystallization on a Wettability Gradient Surface, Langmuir, 28 (2012) 3174-3179. Copyright (2012) American Chemical Society). [79]

Figure 9 - ATR-FTIR imaging of lysozyme protein crystals formed on the Si ATR element. Visible image is shown on the right panel and the OTS gradient along the imaging surface is shown as a plot below the FTIR image. (Reprinted with permission from S. Glassford, K.L.A. Chan, B. Byrne and S.G. Kazarian, Chemical Imaging of Protein Adsorption and Crystallization on a Wettability Gradient Surface, Langmuir, 28 (2012) 3174-3179. Copyright (2012) American Chemical Society). [79].

Figure 10 - Schematic diagram on Micro ATR FTIR imaging optics with hanging drop protein crystallization experiment in place. (Reprinted from S.E. Glassford, L. Govada, N.E. Chayen, B. Byrne and S.G. Kazarian, Micro ATR FTIR imaging of hanging drop protein crystallisation, Vibrational Spectroscopy, 63 (2012) 492-498, with permission from Elsevier). [87] 
Figure 1

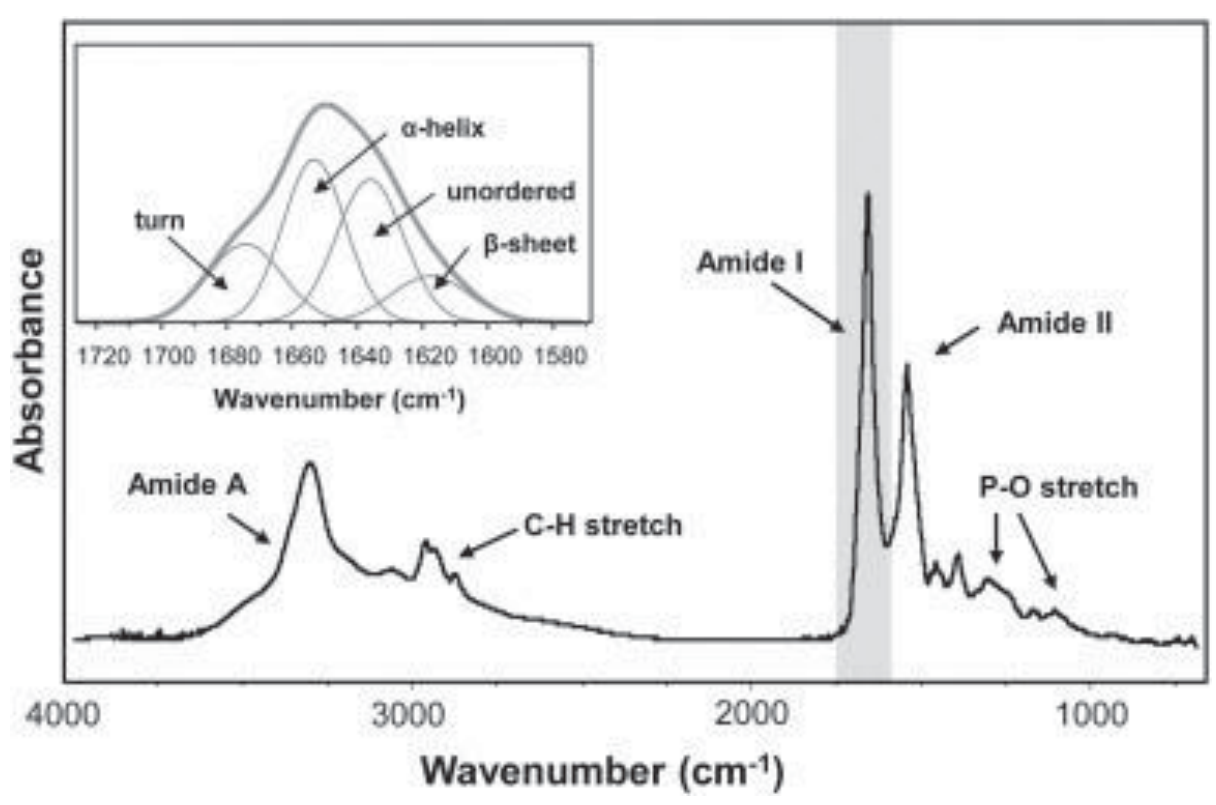




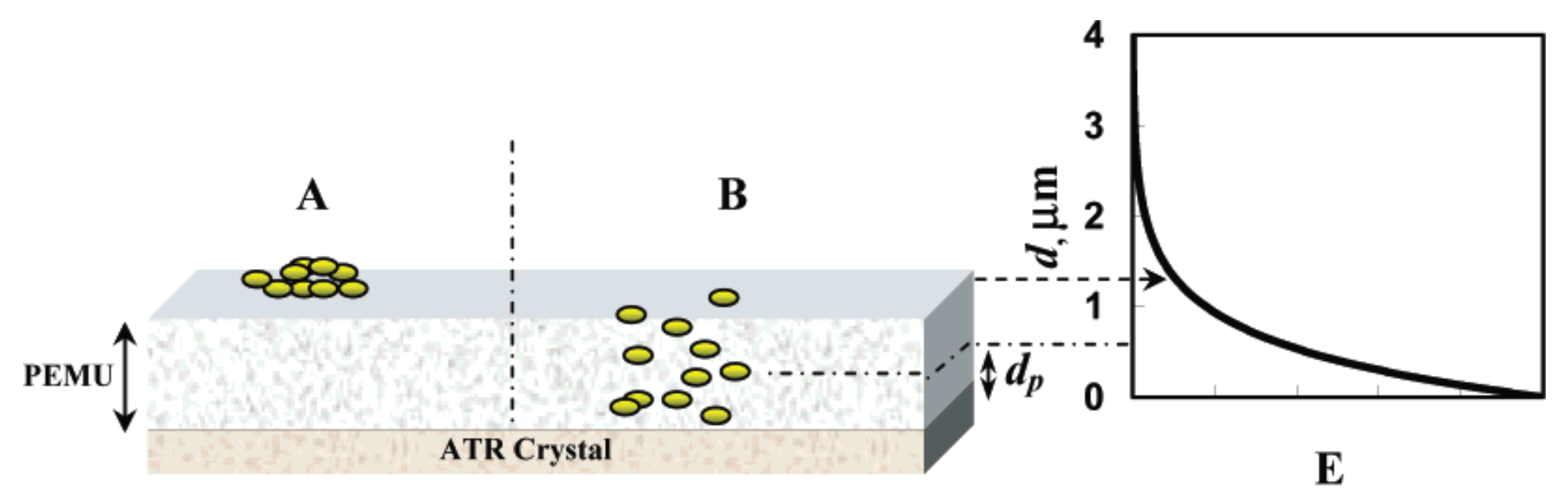




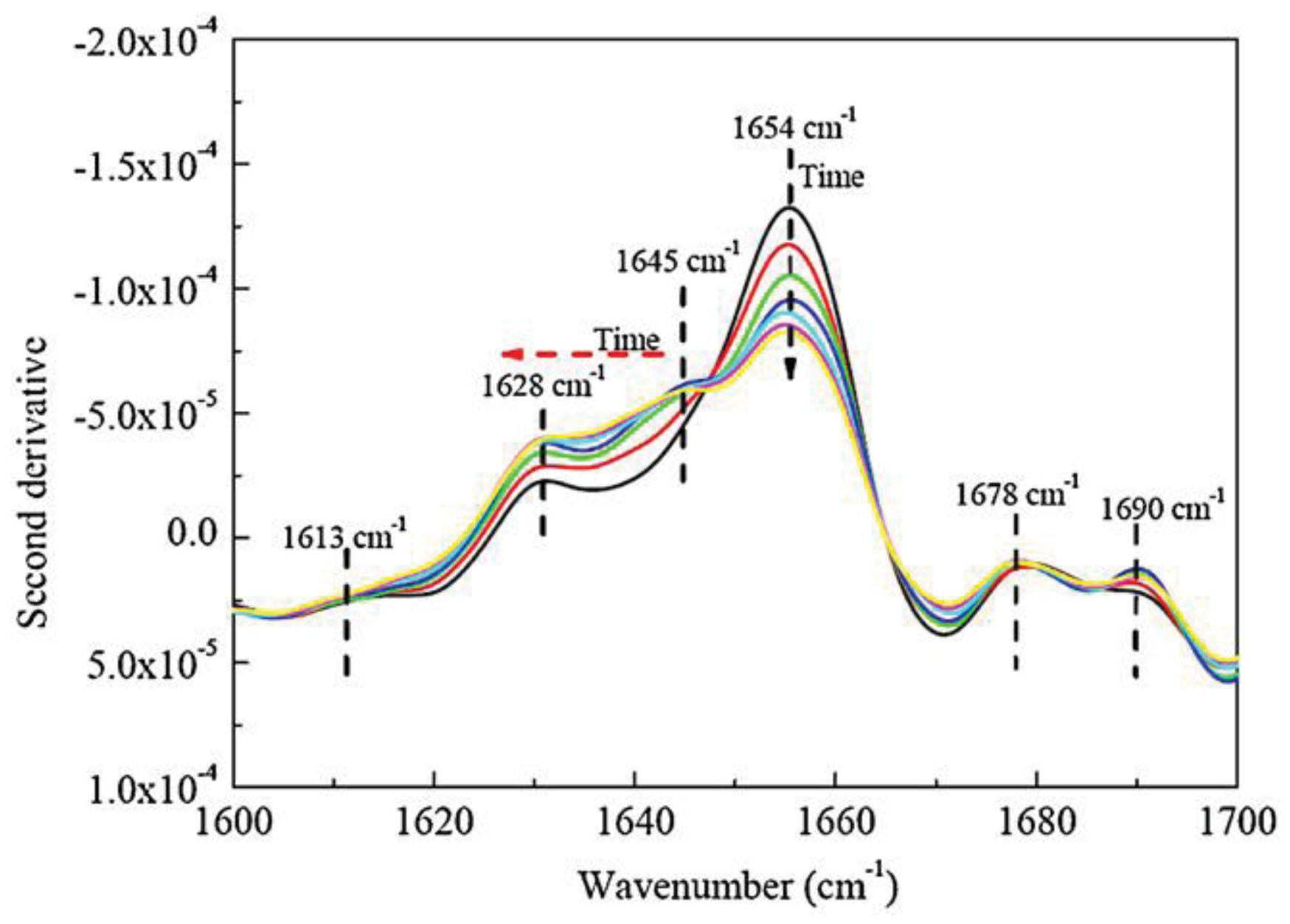




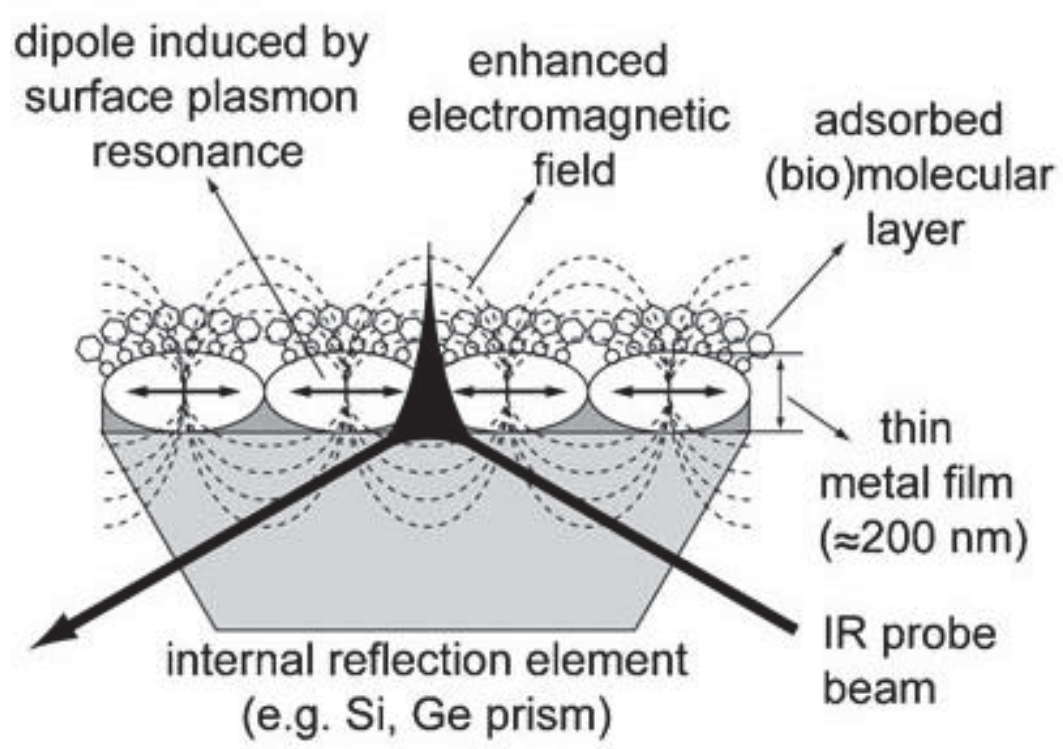




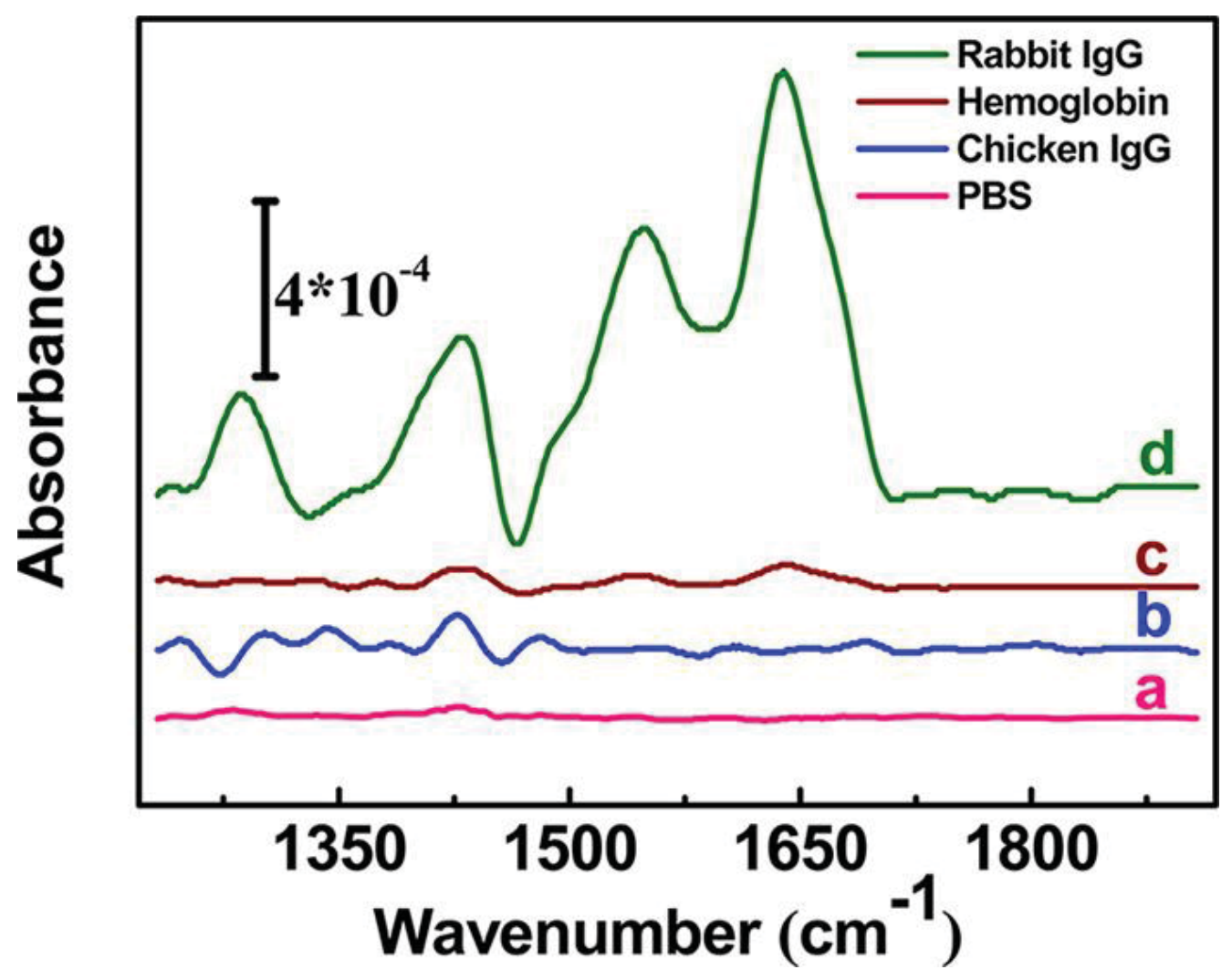


Figure 6

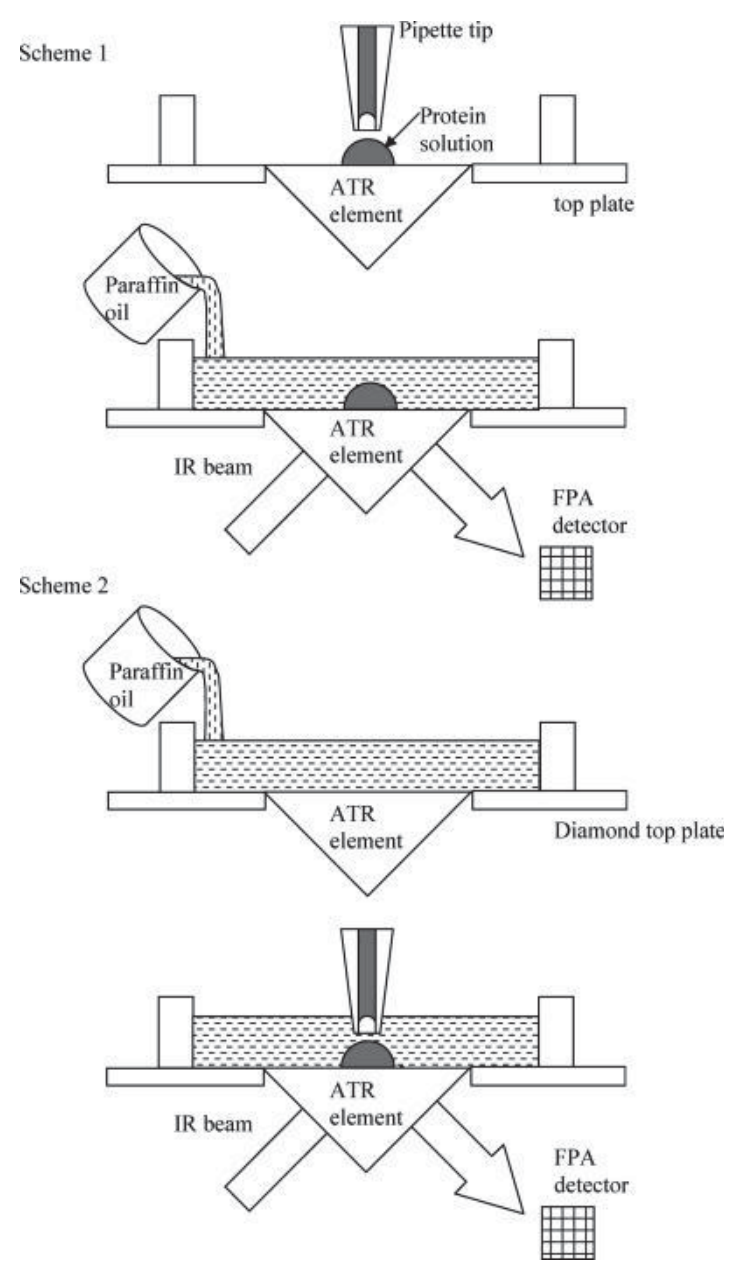


Figure 7

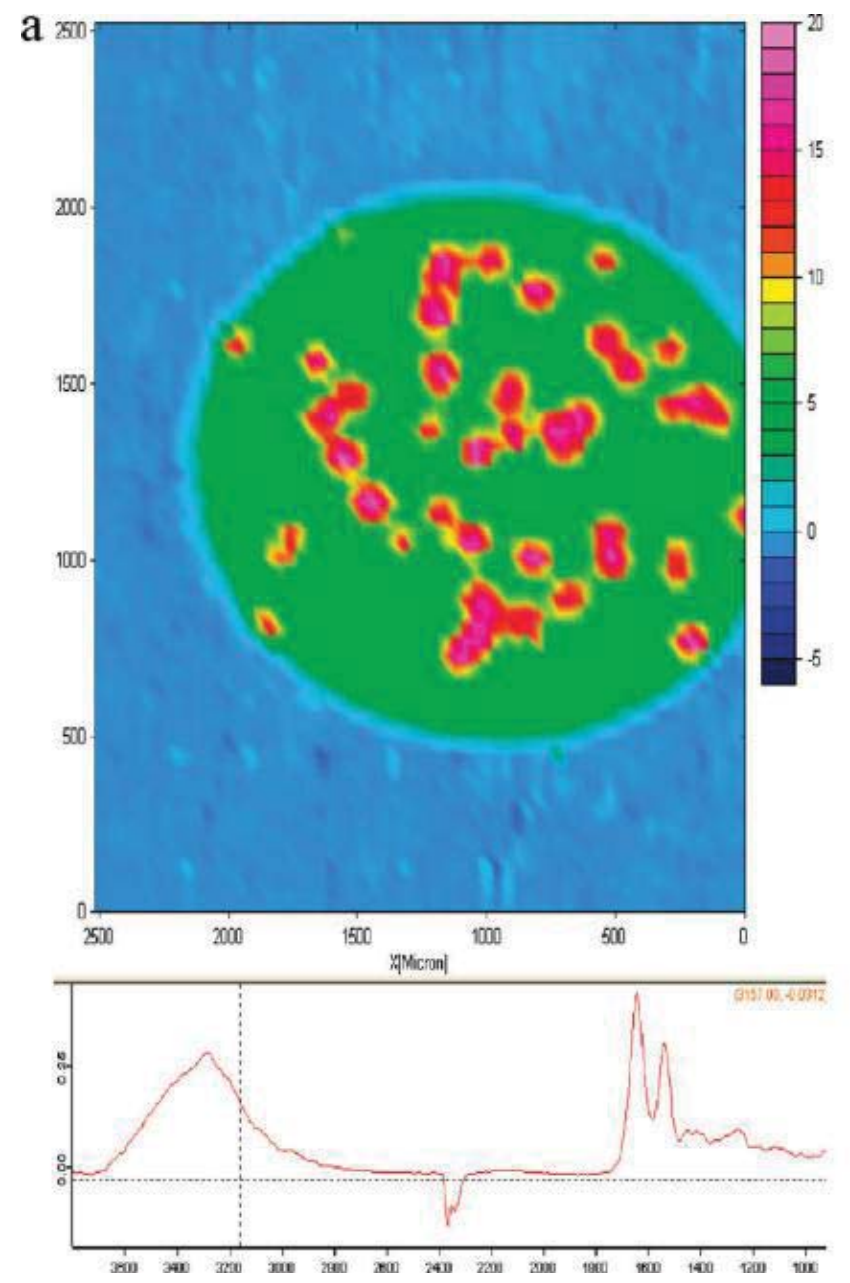

b

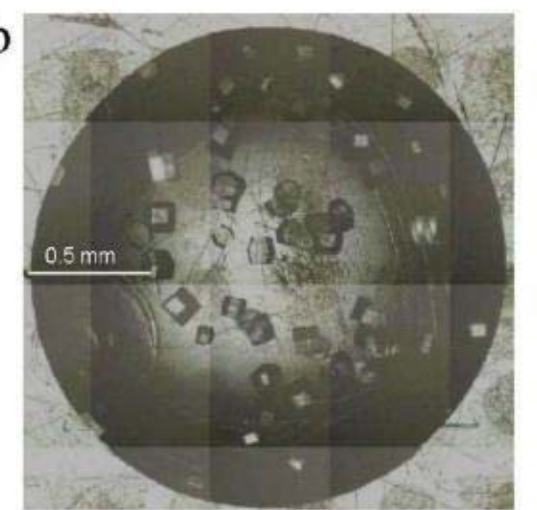

c

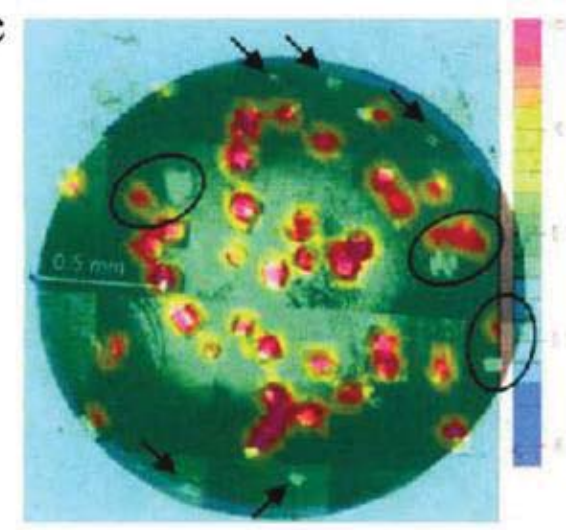




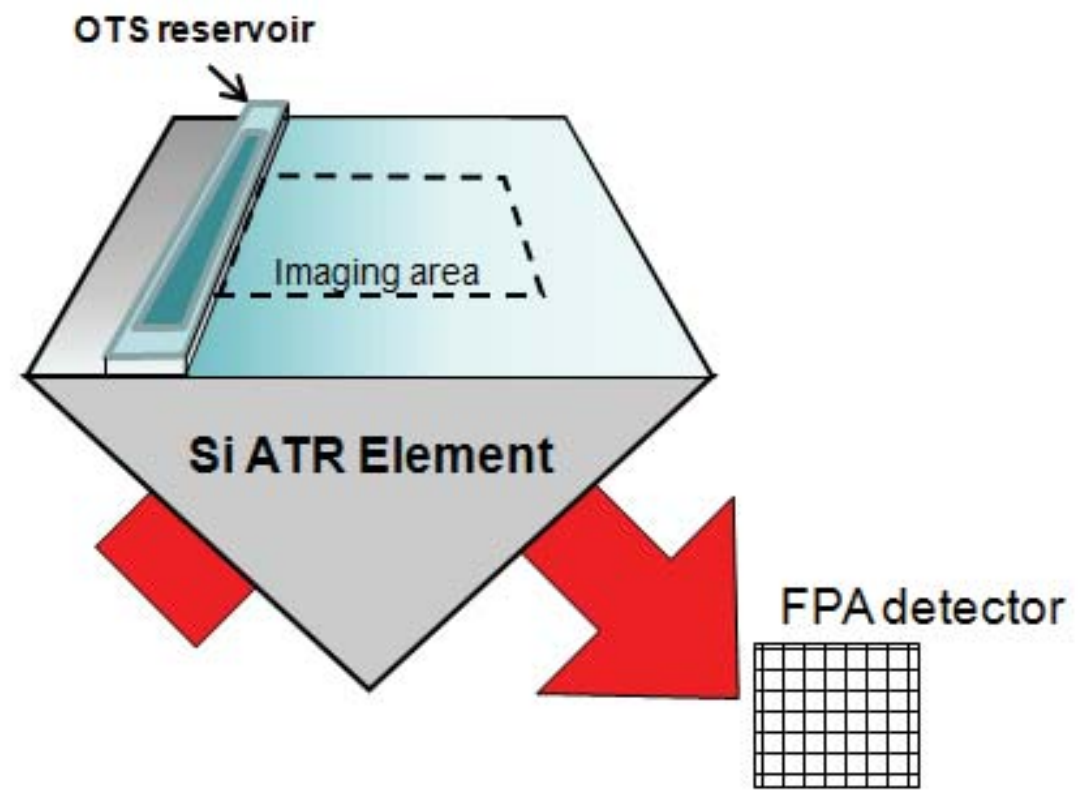


Figure 9 Increasing hydrophobicity
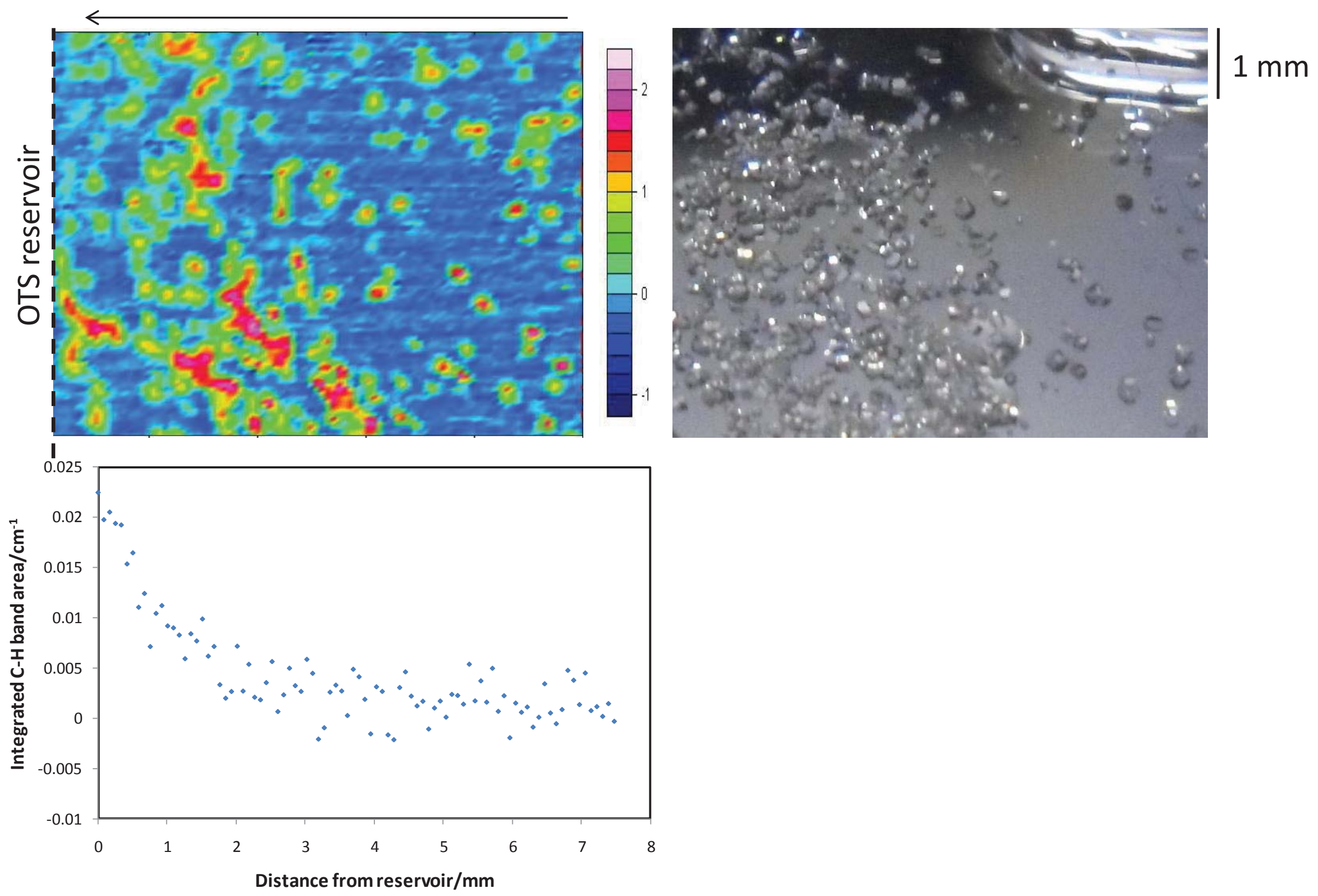


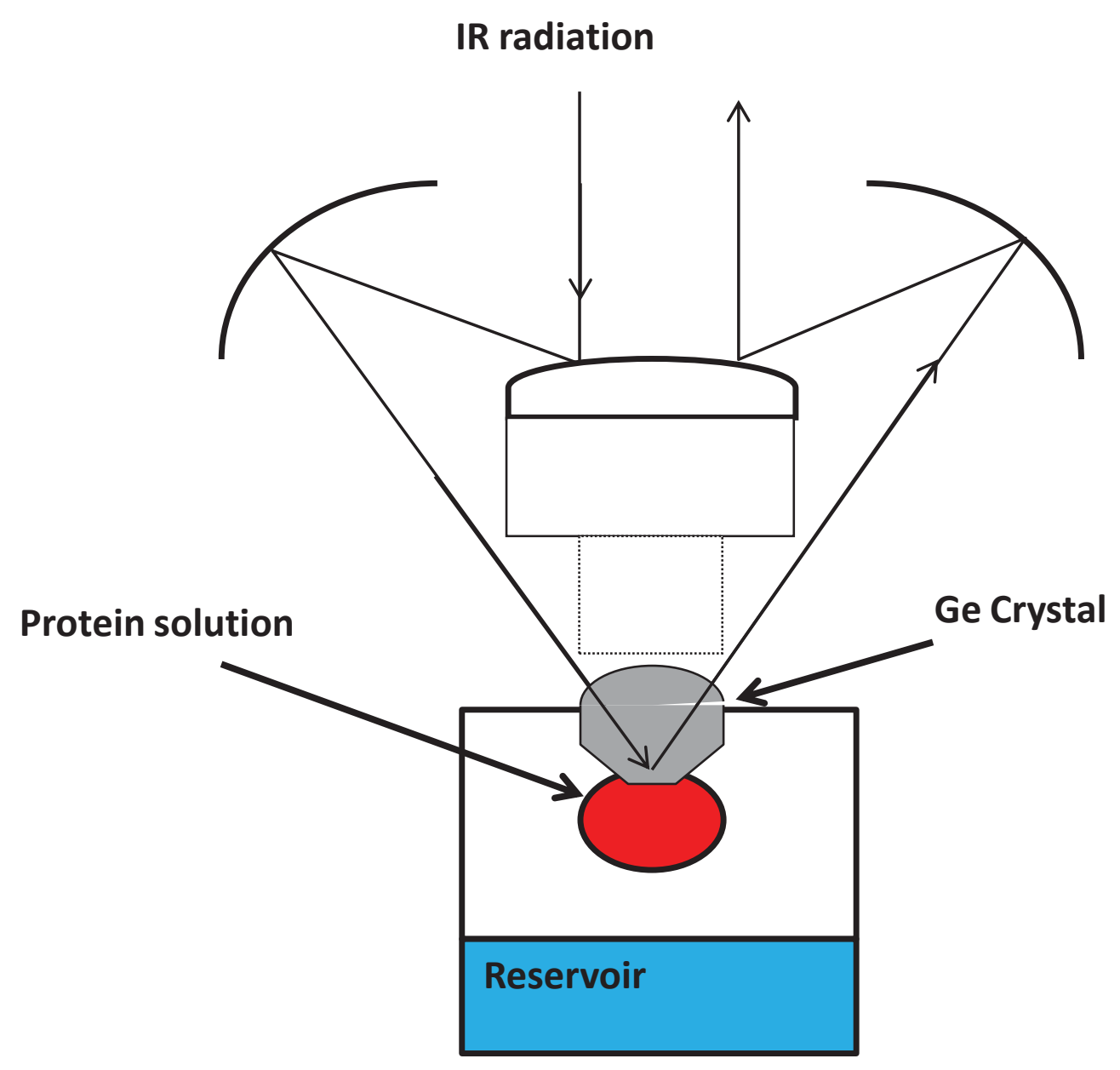

